\title{
MDDQL: A VISUAL QUERY LANGUAGE FOR METADATA DRIVEN QUERYING
}

\author{
E. Kapetanios, M. C. Norrie, D. Fuhrer-Stakic \\ Institute for Information Systems \\ Swiss Federal Institute of Technology (ETH) \\ CH-8092, Zurich, Switzerland \\ kapetanios@inf.ethz.ch
}

\begin{abstract}
We present a meta-data driven query language (MDDQL) as a visual query language. The query language has been specified in such a way that interpretations of data values, attributes and schemas are explicitly taken into account during query construction. These can be expressed directly in the structure of MDDQL terms which are represented as objects within a meta-data or ontological database. They are mainly classified into two categories: domain of interest and operations. In order to alleviate the task of query construction when large and, somehow, difficult to understand conceptual schemas and value domains are considered, the construction of the intended queries is done incrementally on a Web-based blackboard used as a visual query interface through system guidance. Therefore, query construction becomes a matter of moving amongst consistent query states. Inferences about a potential query consistent state to move onto are made by the query language interpreter which implements a kind of state automaton. The inferences are based on the background knowledge, as represented in terms of MDDQL term objects and their connectionism issues forming a cyclic directed graph, and the context of the current query state. Each consistent query state includes a subset of MDDQL terms as inferred by the language interpreter. These might refer to complex or more abstract terms given the recursively defined structures of MDDQL terms. This is particularly useful when large schemas and/or well-restricted value domains are addressed.
\end{abstract}

Keywords: Meta-data, Knowledge representation, Finite State Automata, Visual Query Languages and Systems

\section{INTRODUCTION}

Querying a database managed by a Data Base Management System (DBMS) is usually done by special purpose languages, called query languages, which 
are defined as mappings from a Universe of Discourse into a subset of it, which is expressed by the query result. However, the most widely used database query languages require knowledge about language syntax, information and full understanding of the application domain. All these query languages do not directly address the meaning of the data, when a query is being constructed. This is particularly crucial when large or complex database schemas are queried by end-users which are no experts in a specific application domain, or they are not willing to understand the underlying data model and/or the interpretation of data values as stored in a DBMS.

Consider, for example, two application paradigms: a) a Regional Avalanche Information and Forecasting System - RAIFoS which is concerned with the collection and querying of a large number (ca. 60-80) of physical parameters, b) a Mines Information System - MinesIS which is concerned with more than 200 attributes organised within a data model referring to dissimenation of various kinds of explosive devices over regions involved in war.

In both cases, besides the difficulties of coping with the semantics of a database model or schema, addressing particular values for conditional statements of queries requires a thorough knowledge of well-restricted value domains, such as \{southern slopes, eastern slopes, northern slopes, unknown exposition, no avalanche, in various or all expositions, wind protected slope $\}$ referring to exposition of avalanche as an attribute. Additionally, they are all encoded in the storage model such as $\{0,1,2,3, \ldots\}$, respectively. In the following, we refer to all these symbols - including symbols for database schema elements such as attributes or relations/classes - standing for something else as implementation symbols.

Despite the fact that visual query systems and/or languages are a step towards an end-user friendly way of querying a database in that query language syntax is avoided, the embedding of meaning and/or interpretation of data during query construction is not considered. Thus formulation of a query requires interpretation not only of the data model, but also of the values themselves to be considered for conditional statements. Moreover, query construction through graphical representation of entire conceptual models turns out to be overwhelming, especially, when large conceptual schemas are considered. In addition, no reference to well-restricted value domains is made at the level of conceptual schemas.

In order to alleviate the task of formulating a semantically well-defined query without knowledge about language syntax and with no need of database schema and values interpretation, we elaborated a Web-based Visual Query System, which provides user interaction based on a Meta-Data Driven Query Language - MDDQL with a graph-based visual formalism. The alphabet of MDDQL consists of two main subsets: a) Domain of Interest terms, b) terms standing for operations. Domain of Interest terms refer not only to conceptual 
schema elements but also to values as elements of sets of well-restricted value domains.

Since the terms of MDDQL are a means of incorporating interpretation of implementation symbols, they are conceived as objects having attributes which refer to natural language words and/or annotated descriptions. Therefore, query construction is done on the basis of a semantic triangle as given by the triple <term, word, symbol>. However, in order to minimise the query construction effort, the incremental query construction process takes place in terms of system suggestions which are inferred on the basis of the current query context and the background knowledge as represented by the structure of the terms as well as their connectionism.

Given that preconditions are also encountered for well-restricted value domains, semantic constraints also apply to the consideration of conditional values of the intended query. Similarly, operations are assigned to query constructing elements on the basis of semantic constraints.

The construction of the query takes place on a web-based blackboard as a visual query interface of MDDQL and relies on a WIMP (Window, Icon, Menu, Pointer) user interface. Windows are used as containers for the presentation of a set of terms to be suggested to the user due to the current state of query construction. Icons might accompany the visual representation of terms, such as images referring to particular terms from the set of data values or symbols for operations. A set of suggested terms is presented to the end user when a particular query term is activated. Entry points are any initial terms which address main concepts as a minimal subset of all potential concepts.

Background and related work. The importance of avoiding an underlying query language formalism when end-users need to pose queries to a database system has received much attention during the last 10-15 years within the database research community and many Visual Query Systems (VQS) and/or Visual Query Languages (VQL) have been developed to alleviate the end-users' tasks. A survey of these approaches is given in (Catarci et al., 1997).

VQSs can be seen as an evolution of query languages adopted in database management systems in order to improve the effectiveness of Human-Computer Interaction (HCI). Thus, their most important features are those that determine the nature of the human-computer dialogue, in order to maximise user task performance (Chan et al., 1998). They mainly rely on the integration of the data model and query language in a user-database interface (Chan, 1997) as well as presentation and interaction components that together form a graphical user interface (Murray et al., 1998a).

In particular, query languages based on visual formalisms have been proposed (Cardiff et al., 1997; Florescu et al., 1996; Papantonakis and King, 
1995; Merz and King, 1994; Meoevoli et al., 1994; Clark and Wu, 1994; Haw et al., 1994; Berztiss, 1993; Ozsoyoglou and Wang, 1993; Siau et al., 1992) in order to tackle the problem of query construction. In all these approaches, the conceptual or implementation model is mainly integrated into the query paradig$\mathrm{m}$. However, query construction takes place in terms of navigation through a graphical representation of the entire model and without any consideration of semantic constraints such as posed by well-restricted domain values and/or by their interpretation, mutually exclusive properties and/or values, etc.

This is also not the case for graph based visual formalisms for both ObjectOriented DBMSs where traversal paths can be expressed as queries (Yu and Meng, 1998; Chavda and Wood, 1997), and for a Web query system (Li and Shim, 1998), where a visual user interface - WebIFQ (Web In-Frame-Query) - is used to assist users in specifying queries and visualising query criteria including document meta-data, structures, and linkage information.

Traversal like approaches using graph queries or high level concepts also underly the development of query interfaces for large clinical databases (Liepins et al., 1998; Taira et al., 1996; Hripcsak et al., 1996; Banhart and Klaeren, 1995), or for general purpose systems (Chu et al., 1993; Doan et al., 1995; Chu et al., 1996; Murray et al., 1998a; Murray et al., 1998b; Gil et al., 1999; Zhang et al., 1999). The query generators mostly use an object-oriented data model or functional models such as in case of (Gil et al., 1999).

Despite the fact that in all these approaches query construction is done by navigational issues where the end user does not need to learn a particular query language, it is very often hard to operate on complex or large diagrammatic representations, especially when large database schemas must be considered. Furthermore, the query elements are not inferred on the basis of semantic constraints which hold amongst attributes, well-restricted value domains and/or operations, which might lead to semantically incorrect queries.

Even in cases such as (Gil et al., 1999; Zhang et al., 1999) where data values are considered for the incremental formulation of the final query, these values are not addressed within well-restricted value domains and cannot be subject of a semantically meaningful consideration of values for conditional statements given the current query context. Without such a meaningful consideration of values, queries might be constructed the results of which are, semantically speaking, not worth addressing and might lead to expensive operations without considerable results. In (Gil et al., 1999), where values can be taken from intermediate results during query construction and further refine the final query, we are still faced with the problem of addressing values from pre-calculated results which, in case of large databases, might exceed several hundreds of rows or tuples. 
Organisation of the paper. In this paper, we focus on MDDQL as a Visual Query Language at the core of a Web-based Query Answering System. Section 2 gives an overview of MDDQL in terms of its constituting components, the meta-data database as a repository of MDDQL terms represented as objects, the interpreter realising a kind of state automaton underlying the system guided query construction, and the visual query interface and formalism as a front-end. Section 3 covers the user interaction strategy for the query construction process. In particular, we focus on a scenario referring to the query construction paradigm due to the scientific application domain of RAIFoS. Finally, some formal aspects underlying MDDQL are presented in section 4.

\section{LANGUAGE COMPONENTS AND OVERVIEW}

Figure 1 depicts a general overview of MDDQL as a Web-based, Visual Query Language. It consists of three components: (a) a Visual Query Interface, (b) an Interpreter realising a kind of finite state automaton, and (c) a metadata database, as a repository of MDDQL terms. Since all components are implemented in Java and OMS Java - a Java based realisation of the OM model, an Applet-based version of MDDQL is also available and capable of running within a Web Browser.

As part of a Web-based Query Answering System relying upon a three-tier architecture, all components can be down loaded to the client from an application server at a middle layer where the meta-data database resides. Thus the query construction process takes place locally at the client side.

Before having a closer look at these components, we first depart to some definitions underlying the concepts of MDDQL as a visual query language. We strongly rely on the formal specification of a visual query system given by the triple $M, V, C$, where $M$ refers to internal representation of MDDQL terms (2.1), $V$ to the views called query nodes (2.2), and $C$ to the visual presentation of query nodes (2.3).

\subsection{THE META-DATA DATABASE}

An MDDQL term is conceived as a unit of thought and is represented as an object which is assigned a unique identifier. A term might be associated with one or more words. Furthermore, a term is associated with implementation symbols which stand for storage encodings. Therefore, we specify a query semiotics triangle built up from terms, words and implementation symbols. An implementation symbol is any kind of symbol used for the internal storage of values and/or attributes, whereas a word is a natural language element.

For example, the terms OM600, OM601, OM602, OM603, OM604, OM605 as part of the domain of interest are assigned the words radiation, temperature, 


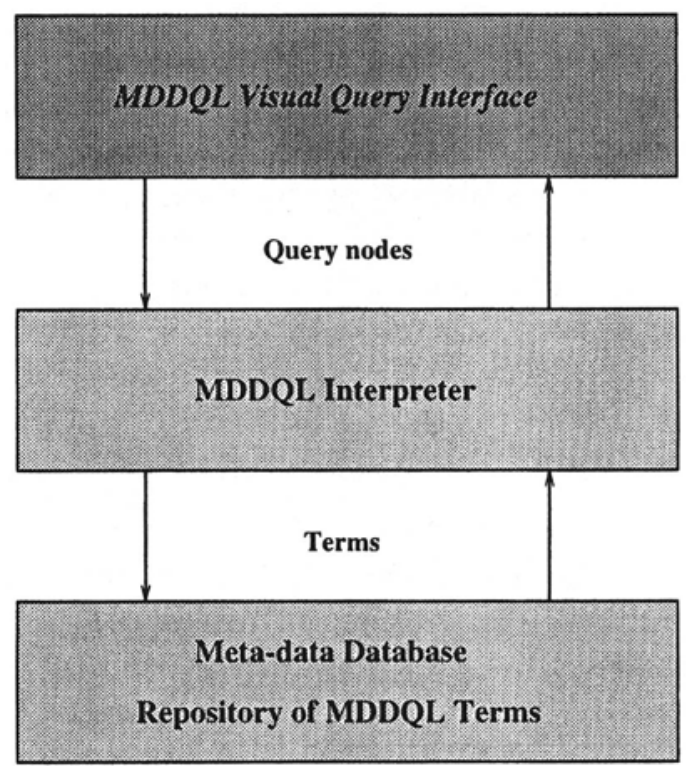

Figure 1 MDDQL components

exposition, northern slopes, eastern slopes, no avalanche, respectively. They might also be assigned the words Strahlung, Temperatur, Lage, Nordhang, Osthang, keine Lawine, respectively. On the other side, the terms are assigned the implementation symbols $R W, T A, L A, 2,1,0$. Note that the three last terms for$\mathrm{m}$ a well-restricted value domain for the property exposition(English) or Lage (German). Additionally, the arithmetic intervals $[0,120]$ and $[-50,50]$ are also represented as terms which stand for well-restricted value domains referring to radiation (OM600), temperature (OM601), respectively.

Thus it is possible to assign words in different languages to the same term and still keep on referring to the same implementation symbol. This enables querying by using elements of different natural languages without any side effects upon the query results. The set of all MDDQL terms will be called alphabet and is mainly divided into two subsets: domain of interest and operations to apply on it. All terms (objects) are managed and provided by a meta-data database the underlying connectionism model of which is a cyclic directed graph connecting terms, which belong to the subset of domain of interest terms.

Since terms are conceived as objects, additional attributes can provide more characteristic properties for particular terms. For instance, a description attribute can provide a more descriptive piece of information referring to the notion of a term, such as reflection intensity of solar radiation for radiation 
(OM600), or the measurement unit in which an arithmetic interval is expressed as a well-restricted value domain for an attribute, such as watt per square meter for radiation (OM600).

Moreover, terms (objects) are assigned a role as members of collections or classes. We mainly distinguish among the roles of concepts, relationships, properties (categorical, numerical), concrete value domains, all of which might be atomic or complex. For instance, the terms OM600, OM601 are classified as properties (numerical) where the term OM602 is classified as properties (categorical), since the assigned well-restricted value domain is an arithmetic interval.

Analogously, operations such as $O M 700, O M 701, O M 702, O M 703, O M 704$ are assigned the natural words minimum, maximum, absolute frequency, distribution, mean value and might also be assigned to implementation symbols such as min, $\max , A F, D i, M V$. Furthermore, they also underly a classification schema such as one-dimensional, two-dimensional, categorical, numerical, etc..

The connectionism model provides three kinds of mappings: a) those holding for terms which are members of the domain of interest but have distinguished classifications - interconnecting links, b) those holding for terms which are members of the domain of interest but belong to the same class - recursive links, c) between collections of domain of interest terms and operations terms. Recursive $N: M$ mappings over the same class of terms enables the formation of more complex elements such as assignment of concrete values to concrete value domains, the construction of complex attributes and/or concepts such as coordinates being a property defined over properties: height, longitude, latitude, or Automatically measured data being a concept defined over concepts: ENET data, IMIS data.

Therefore, construction of a query becomes a matter of navigation through a so-called query information space $Q I S$ as formed by all terms of MDDQL. $Q I S$ is conceived as an cyclic directed graph $G_{Q}$ which consists of $(V, E)$ defined as $V=\left\{v_{n} \mid n \in N\right\}$, where $V$ is the set of vertices standing for terms, and $E=\left\{\left(v_{n-1}, v_{n}\right) \mid v_{n-1}\right.$ is a parent of $\left.v_{n}\right\}$, where $E$ is the set of directed edges standing for the mappings among terms. $V_{I}$ would be the set of entry vertices without any incoming edges.

\subsection{MDDQL INTERPRETER}

Since query construction takes place without any knowledge of language syntax, the user is guided by the system in order to pose her/his intended query. This is achieved by moving through consistent query states when the current query state is given. Thus the inference of which subsequent query states are consistent with the current one is provided by the MDDQL-Interpreter on the basis of a formally specified state automaton. 
Definition:. A query state $S_{q}$ is a set of nodes such that $S_{q} \subseteq V$. An initial query state $S_{q_{0}}$ is a set of initial nodes $S_{q_{0}} \subseteq V_{I}$

Definition:- For a given query state $S_{q_{n}}$, the set of candidate terms $C_{n}$ is defined as $C_{n}=\left\{v_{j} \mid\right.$ for some $v_{i} \in S_{q_{n}},\left(v_{i}, v_{j}\right) \in v$ and $\left.v_{j} \notin S_{q_{n}}\right\}$

Definition:- A precondition is a formula $p_{v_{m}} \in P_{V}$ in conjunctive (AND-connected) or disjunctive (OR-connected) normal form connecting vertices (terms) $v_{i} \in V$, where $P_{V}$ is the set of all preconditions associated with particular vertices $v_{m} \in V$. Let us call $p_{v_{m}}^{\prime}$ the set of vertices appearing in $p_{v_{m}}$, it holds that $p_{v_{m}} \rightarrow$ true, if $\exists v_{i} \in p_{v_{m}}^{\prime}$, such that $v_{i} \in S_{q_{n}}$ for disjunctive preconditions, or $\forall v_{i} \in p_{v_{n}}^{\prime}, v_{i} \in S_{q_{n}}$ for conjunctive preconditions.

Definition:- For a given query state $S_{q_{n}}$, a set of candidate terms $C_{n}$ is consistent, if for each $v_{j} \in C_{n}$, either there is no precondition $p$ associated with $v_{j}$ or $p$ evaluates to false for the given query state $S_{q_{n}}$.

Definition:- For a given query state $S_{q_{n}}$, a potential query state $S_{q_{n+1}}$ is $S_{q_{n}} \cup C_{n}$, where $C_{n}$ is a consistent set of candidate terms for $S_{q_{n}}$.

However, the set of consistent candidate terms is being inferred each time a particular term is activated by the end user. This means that the end user is interested in extending and or refining her/his query. The communication between Interpreter and Visual Query Interface takes place in terms of interchanging objects which are representing query nodes. These are constructed and provided by the interpreter to the visual query interface.

The query nodes correspond to the views of terms. They include a subset of the available information concerning terms. Currently, it is restricted to slots such as

OID, label (word), description, connector, role, operation, link label, link type

where label refers to the natural language word, description refers to term annotations, role to the role of term due to the classification model mentioned in 2.1, connector to the logical connector (AND, OR, NOT), link label to the kind of link (IS-A, part-of, etc.), link type to the notion of link such as assertional or definitional. Depending on the case, some of these slots have to be initialised by the selections made by the end user. For instance, name when an arithmetic interval or value has to be set, or operation when a particular operation is assigned out of a set of semantically consistent operations (see also section 3.2). 


\subsection{THE VISUAL QUERY INTERFACE}

The query construction takes place on a blackboard and relies upon a graphbased notation. Each query node is represented by a rectangle in different colours or an elliptical circle denoting the role of the term (query node) within the query such as concept, relationship, property, or specific value. Figure 2 gives an example of a query expressed by MDDQL elements. Representation of the query nodes is based upon the contents of the query nodes as objects delivered by the interpreter.

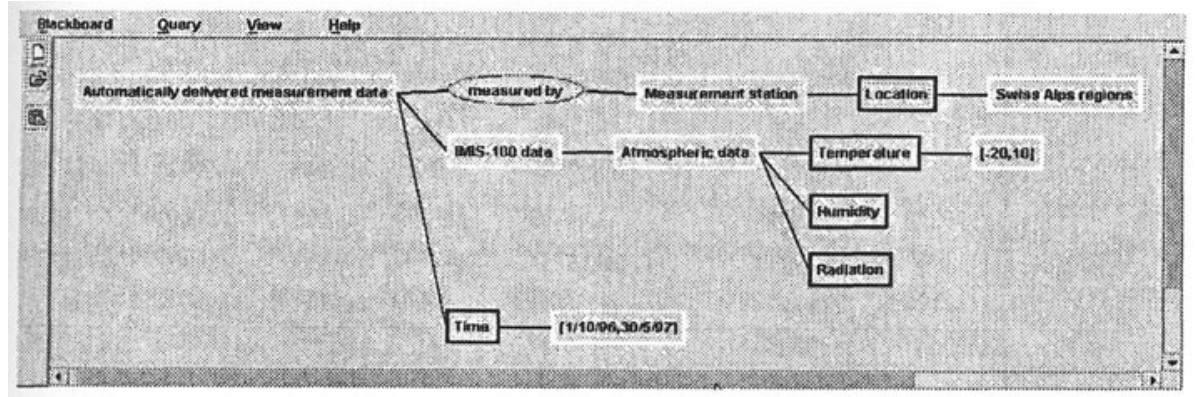

Figure 2 An example query expressed in MDDQL terms

Nodes are labelled by assigned words of a specific natural language - in our example English - and are linked with other nodes constituting a query graph. Links can be labelled optionally indicating the notion of the link by using, in turn, specific natural language words such as is-a/as, composed of, characterised by, etc. Additionally, annotations of terms can be viewed by pointing the cursor on a particular term. On the other side, operations are assigned to nodes by using specific icons (more details are given in 3.2).

Since operations are also subject of semantic inferences, they must be selected out of a set of suggested operations which are semantically consistent with classes of nodes or terms, they are all presented by their language language counterpart. Therefore, the operation symbols are only indicative of which terms have been assigned operations. Logical connectors (AND, OR, NOT) are also foreseen and are separated from the other operators. Nodes standing for values can be negated by turning them to dark shaded rectangles.

\section{INTERACTION STRATEGY}

For each query construction session, the user addresses the corresponding URL for a particular application domain. An initialised Applet is down loaded 
which includes the Java packages for the visual formalism as implemented with the Swing library, Additionally, the Java package for the MDDQL Interpreter and the OMS Java files for the meta-data schema providing the representational issues of MDDQL-terms together with the instances (terms) themselves, which correspond to the relevant application domain are also down loaded to the client site. Therefore, the first two front-end layers (figure 1) together with a copy of the meta-data database for MDDQL-terms are available in the main memory of the client's machine. In the following scenario describing the user interaction strategy for query construction, we distinguish between construction issues referring to terms of the Domain of Interest (3.1) and those referring to Operations (3.2).

\subsection{VIEWING THE DOMAIN OF INTEREST}

At the beginning of query construction session, the user is requested to choose a preferred natural language in which the annotated nodes and links, as well as additional elements, are going to appear. For the sake of convenience, we will restrict ourselves to English as a presentation language in the following examples, and to paradigms which refer to the application domain of RAIFoS. We will also refer to terms by using the corresponding natural word and not the object identifier, when possible, since internal representation issues of terms are hidden from the end user.

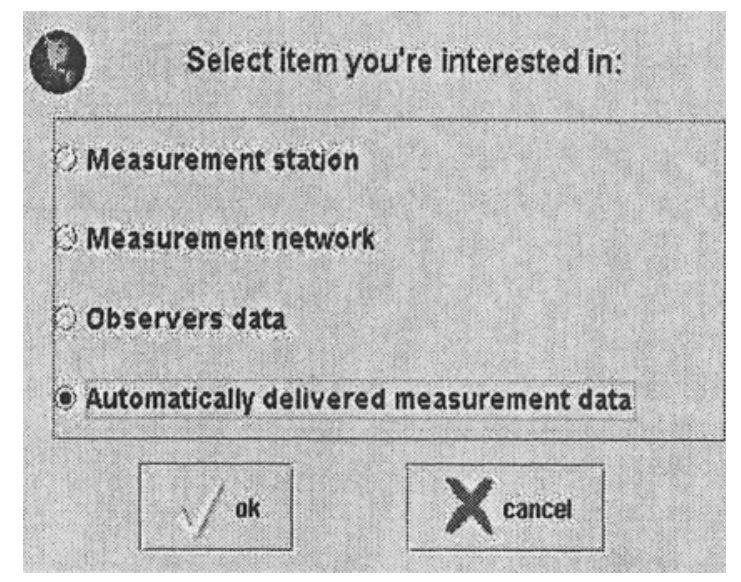

Figure 3 A proposed set of MDDQL terms to start with

Assuming that the end user either has no idea or does not need to gain some knowledge about the underlying database issues, she/he is required to choose an initial term out of the set of initial terms $V_{I}$ as provided by the 
system (MDDQL-Interpreter). This puts the emphasis on the main subject, a concept with which we would like to start constructing a query. For example, an initial set of terms could have been \{Automatically delivered measurement data, Observers data, measurement network, measurement station\} (figure 3). If the term Automatically delivered measurement data would have been chosen, a query node is constructed out of the terms, as explained in section 2.1 , and positioned on the blackboard.

In order to move further towards query refinements and/or restrictions, the user has to click on a query node. Consequently, the system (MDDQL-Interpreter) comes up with a set of potential query nodes (terms which are presented within a popped-up selection window). One or more of the suggested terms might be selected and, therefore, linked with the clicked node either (a) as being a special case of a previous term (query node) - recursive links, e.g., ENET 10-minutes data, ENET 1-hour data, IMIS-100 data might be connected to automatically delivered measurement data, or (b) as assigned characteristics through interconnecting links such as properties, for instance time, characterising a particular concept or relationships, for instance measured by, leading to other concepts (see also figure 4).

Let us assume that the user wishes to further refine her/his query in that IMIS-100 data further refines the current query state automatically delivered measurement data $\}$ which now becomes $\{$ automatically delivered measurement data, IMIS-100 data\} (checked box in figure 4). A further refinement will be caused by clicking on the query node IMIS-100 data which results in a suggestion consisting of the set of terms $\{$ Wind data, Atmospheric data, Snow data\}, (see also figure 5), since all these kinds of terms are conceived as part of ENET 10-minutes data, ENET 1-hour data or IMIS-100 data.

Selecting Atmospheric data as a term to extend the current query state which now becomes \{ automatically delivered measurement data, IMIS-100 data, Atmospheric data $\}$, a further query refinement could be done by clicking on the query node Atmospheric data. The set of consistent potential terms would be measured by, Time, Radiation, Humidity, Temperature (see also figure 5). Note that the terms Time, measured by are suggested at both query extension stages (figures 4 and 5), since they are relevant to both activated query nodes IMIS-100 data and Atmospheric data. This corresponds to the internal representational model for MDDQL terms (see also section 2.1) which enables multiple inheritance.

Having selected time or measured by at a particular stage, these terms will not be further suggested within a particular inheritance hierarchy, indicating the fact that they are all relevant regardless the refinement degree of a particular concept. Further semantic constraints also apply to the suggestion of properties when they are inferred by the MDDQL-Interpreter as a consistent set of potential 


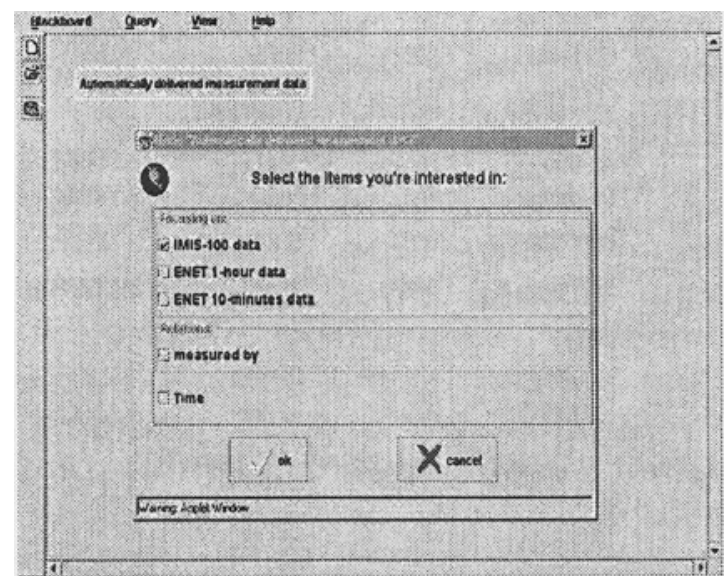

Figure 4 An extension of current query state by adding the term IMIS-100 data

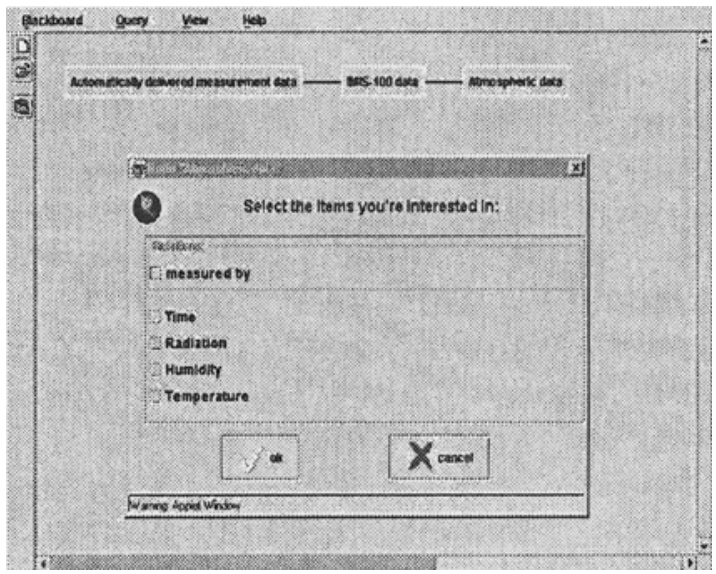

Figure 5 A potential extension of current query state by activating the node Atmospheric data

nodes (terms) (see also section 2.2) to be further considered for the extension of the current query state or graph.

For instance, the properties Radiation, Humidity would not have been suggested, if one of ENET 10-minutes data, ENET 1-hour data had been considered within the current query state, since it makes no sense to address these properties in conjunction with these concepts. Even in case of re-activation 
of the query node automatically delivered measurement data, the term IMIS100 data will not appear within the set of potential terms, since it is already considered within the current query state.

Selected terms extend the current state of the query graph in that all selected terms are positioned on the blackboard by drawing the appropriate links among them. This user-system interaction principle can be applied to all $n$ odes (terms) belonging to the current state of the query graph. For instance, selecting the terms temperature, humidity, radiation will extend the current query state to \{ automatically delivered measurement data, IMIS-100 data, Atmospheric data, Temperature, Humidity, Radiation $\}$. Clicking on node temperature will provide the term $[-50,50]$ celsius as a well-restricted value domain within which conditional values have to be specified (see also figure 6). Any values specified outside this particular range of values will be rejected.

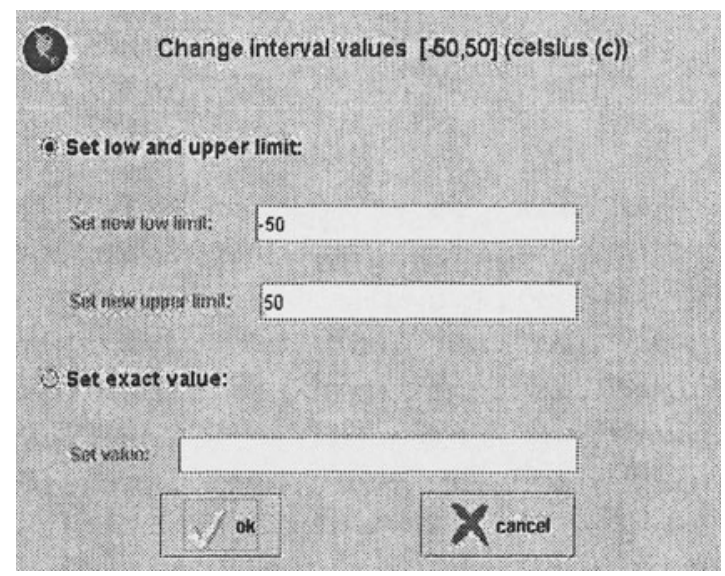

Figure 6 A well-restricted value domain for arithmetic values

Similar restrictions also apply to almost all kinds of well-restricted or concrete value domains. For example, the property direction of wind is assigned two possible well-restricted value domains which should be considered for the intended query, such as $[0,360]$ degrees or the set of categorical values $\{$ North, North-East, North-West, South,... $\}$ within another query context. The arithmetic interval will be suggested by the MDDQL-Interpreter only if the current query state is something like \{Automatically delivered measurement data, ENET 10-minutes data, Wind data, Direction $\}$. The set of categorical values will be presented, as depicted in figure 7 , only when the current query context would have been $\{$ Observers data, Wind data, Direction $\}$. 


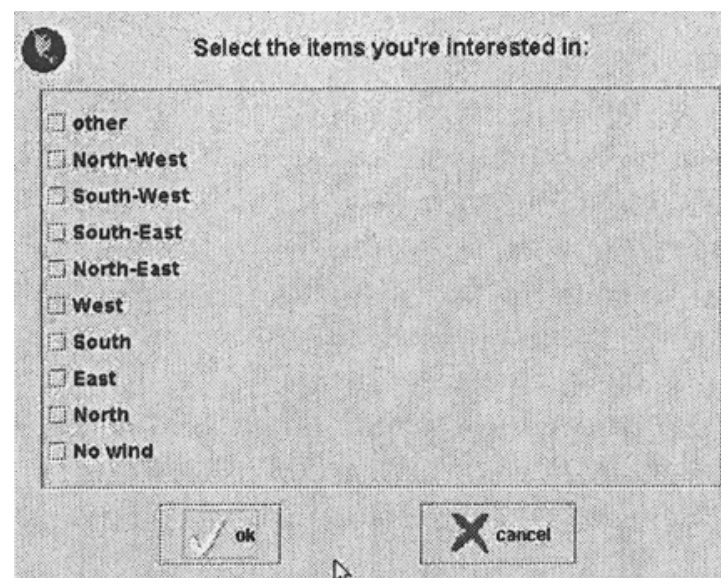

Figure 7 A well-restricted domain with categorical values

A further interaction issue is the possibility of activating the labelling of links which gives an insight into the connectionism semantics of the query nodes (terms). For example, the query state \{Automatically delivered measurement data, IMIS-100 data, Humidity, Radiation, Temperature, $[-20,10]\}-$ assuming the user specified $[-20,10]$ as value constraint for temperature - can be turned into \{Automatically delivered measurement data, as, IMIS-100 data, as Atmospheric data, Characterised by, Humidity, Characterised by, Radiation, Characterised by, Temperature, Constrained by, $[-20,10]$ celsius $\}$. It is also possible to provide a more detailed description of each node (term) by pointing the cursor on the relevant term either on the blackboard or within the popped-up window with the suggested terms, just before a particular term is selected.

Thus it could be possible to gain more information about a particular term before considering it further for the intended query. For instance, the term Radiation is annotated by The reflected radiation from snow, providing an additional explanation of the notion of this term. Similarly, measurement units are also provided as annotations for arithmetic intervals, in order to illustrate the fact that the arithmetic values are expressed in a specific measurement unit (figure 6).

Since further restrictions can be included in terms of relationships associating concepts, as suggested by the MDDQL-Interpreter at a current query state, the natural language based interpretation of the intended query, as depicted in figure 2, would be: 
Get the humidity and radiation of the atmosphere as part of IMIS-100 data, as part of automatically delivered measurement data, where the temperature is within $[-20,10]$ Celsius and are measured by measurement stations located at Swiss Alps region and for the time between 1-10-96 and 30-5-97.

\subsection{ASSIGNING OPERATIONS TO QUERY NODES}

Having constructed an intended query in terms of nodes which refer to MDDQL terms as members of the set of Domain of Interest, the user might assign operations to particular nodes according to their roles within the query. This can be done by clicking on that node with the right mouse button in order to receive a semantically coherent set of operations which can be assigned to the node. The potential set of operations is also inferred by the MDDQL Interpreter and appear within a popped-up window similar to the suggestion of terms from the domain of interest.

These operational inferences take into account the actual role of an MDDQL term as given by the classification hierarchies underlying the meta-data database model as well as the current query state. Assuming that the node is assigned the role categorical variable indicating the fact that the values correspond to categories such as North, North-West, North-East,... even if they are internally encoded by numerical values such as $0,1,2, \ldots$, no arithmetic operations such as average or mean value, deviation will be suggested by the system for consideration. Similarly, comparison operators such as $>=,<=,<>$ are only suggested for nodes which stand for atomic arithmetic values.

On the other side, two- or three-dimensional operations such as time series, scattering diagrams, histograms are only considered in conjunction with the presence of more than one property within the current query state. Furthermore, preconditions might be assigned to the objects representing operations within the meta-data database. Since operations are objects too, additional attributes might be defined which provide more information about the intended usage of each operation. This can be viewed at the time of selecting a particular operation out of the set of potential operations by pointing the cursor on a candidate term (operation) (see also figure 8).

For example, consider the intended query as depicted in figure 8 . Since the property Radiation in classified as a numerical variable, a set of statistical operations is suggested for selection. Having chosen the average operation, the corresponding node standing for Radiation on the blackboard will be annotated by a graphical symbol of this particular operation. Accordingly, the natural language based interpretation of the intended query would be:

Get the average of atmospheric radiation as part of IMIS-100 data, as part of automatically delivered measurement data, where the humidity is greater 


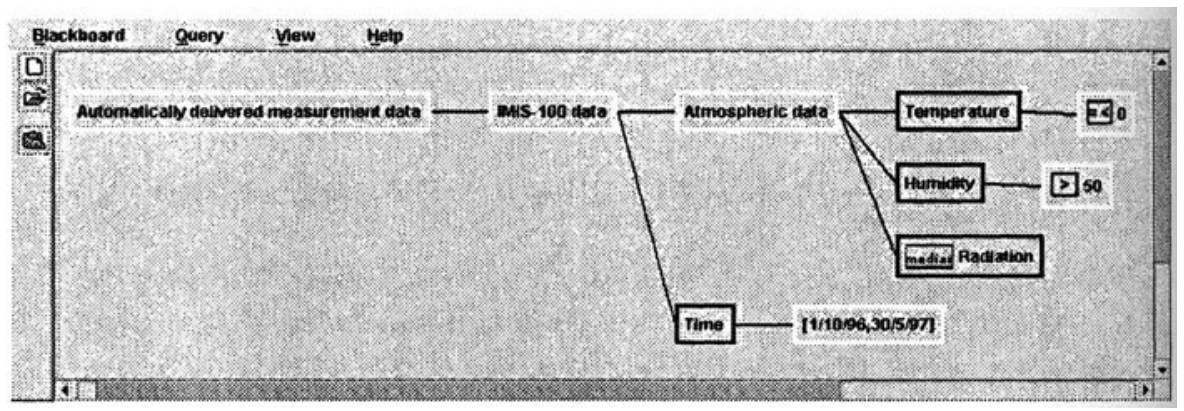

Figure 8 Assigning operations to query nodes

than $50 \%$ and temperature less than or equal to zero (0) Celsius, for the time between 1-10-96 and 30-5-97.

It is also possible to assign logical operators $A N D$ or $O R$ to nodes (terms) classified as concepts or properties as well as negating a term by assigning it the NOT operator. The latter is currently restricted to nodes standing for specified and/or selected atomic or interval values. Consequently, these values should be excluded from the query result.

Finally, the user might submit the constructed query by choosing the execute query option from the query menu of the blackboard. The system will convert the graph based query into a conventional query such as SQL or OQL where internal mappings between the MDDQL terms and implementation symbols are taken into account. In this paper, we will not cover the mapping issues between MDDQL and other database specific query languages as well as the representational issues of query results, since they might be presented by various types such as alpha-numerical and/or graphics, e.g. plottings. We rather focus on the visual query formalism as well as on the query construction mechanism.

\section{FORMAL ASPECTS OF MDDQL}

Most visual query systems or languages, in general, have no underlying formal syntax definitions such as BNF notation for textual query languages, since the bi-dimensionality of such languages exceeds the capabilities of stringbased grammars. The semantics is often operational and expressed by rewriting rules which transform the proposed language into some other well-defined target language.

Since formulation of a query becomes a matter of system guidance on the basis of semantic constraints rather than direct usage of diagrammatic representation of conceptual schemas or other database model abstractions, MDDQL 
falls into the category of generative languages as known to the AI and computational linguistics communities. It this category of languages, the grammar rules are used for the generation of phrases rather than for parsing of already formulated phrases. It is out of the scope of this paper to provide a thorough comparison with widely known generative grammars such as attribute grammars, augmented transition networks, etc.

However, we feel the need to have a formal specification of MDDQL in terms of generative grammar rules in order to gain an insight into the expressiveness of the language as a natural sublanguage, since it is restricted to queries. These rules are a form of production rules, where the symbol $Q$ on the left side must be interpreted as generate query. This determines what to say in addition to the two other stages of how to relate it to the listener or viewer - covered by the visual formalism - and how to map it into a string of words - covered by the chosen vocabulary as provided by the assigned words to terms. All three stages are involved in the genesis of a language.

An overview of these production rules referring to the sequence of terms as members of the Domain of Interest is given in the following:

$Q \rightarrow G Q \mid C Q$

$G Q \rightarrow C O N C E P T$ PROPERTY

$C Q \rightarrow G Q V A L U E-D O M A I N$

$C O N C E P T \rightarrow e\{C O N C E P T|r C O N C E P T| \epsilon\}$

$P R O P E R T Y \rightarrow p\{P R O P E R T Y \mid \epsilon\}$

$V A L U E-D O M A I N \rightarrow c v d\{V A L U E-D O M A I N \mid \epsilon\}$

Due to these production rules, a generated query $Q$ might take the form of a general query $G Q$ or that of a conditioned query $R Q$. A general query constitutes concepts followed by properties, whereas a conditioned query is an extension of a general query in that specific conditions are considered over well-restricted value domains.

Assuming that $E, R, P, C V D$ are subsets of the Domain of Interest which is, in turn, a subset of the MDDQL alphabet, the recursive definition of the last three production rules indicate the fact that: (a) a starting term, as standing for a concept, is an entity $e \in E$ followed by the empty set $\epsilon$ or by a pair of terms standing for a relationship $r \in R$ and entity $e \in E$, respectively, or by an entity $e \in E$, (b) a certain term, as standing for a property, is a property $p \in P$ followed by an empty set or a term standing, in turn, for a property. Finally, a term $c v d \in C V D$ follows a property in a conditioned query where $c v d$ might be followed, in turn, by an empty set or $c v d \in C V D$.

These recursive definitions enable the consideration of complex concepts, properties and/or well-restricted value domains within a query. $e, r, p, c v d$ are not terminal symbols of the grammar but rather variables to be instantiated by the user. A possible alternative would be to assign conditions for applying a rule and/or associate attributes with the non-terminal symbols. This technique 
led to the definition of attribute grammars (Knuth, 1968), where the conditions operate on attribute-value records. However, since the conditions strongly depend on instances (particular terms) and/or the roles of terms, it would be cumbersome or even impossible to express these conditions within the context of production rules.

\section{CONCLUSION}

We presented a meta-data driven query language as a visual query language. End users do not need to make use of a language syntax and/or fully understand the database model, in terms of conceptual schema, attribute and data value interpretations, in order to pose queries to databases, since these interpretations are explicitly embedded within and provided by the MDDQL terms. This also enables the usage of any natural language when queries are constructed.

Additionally, in order to reduce the effort of query construction in terms of large and, somehow, difficult to cope with conceptual schemas, the construction of the intended queries is done by system guidance relying on inferences based on a) background knowledge as expressed by structural and connectionism issues of the MDDQL terms, b) the current query state.

\section{References}

Banhart, F. and Klaeren, H. (1995). A graphical query generator for clinical research databases. METHODS OF INFORMATION IN MEDICINE, 34(4):328-339.

Berztiss, A. T. (1993). The query language vizla. IEEE TRANSACTIONS ON KNOWLEDGE AND DATA ENGINEERING, 5(5):813-825.

Cardiff, J., Catarci, T., and Santucci, G. (1997). Semantic query processing in the VENUS environment. INTERNATIONAL JOURNAL OF COOPERATIVE INFORMATION SYSTEMS, 6(2):151-192.

Catarci, T., Costabile, M. F., Levialdi, S., and Batini, C. (1997). Visual query systems for databases: A survey. JOURNAL OF VISUAL LANGUAGESAND COMPUTING, 8(2):215-260.

Chan, H., Siau, K., and Wei, K. K. (1998). The effect of data model, system and task characteristics on user query performance - An empirical study. DATA BASE FOR ADVANCES IN INFORMATION SYSTEMS, 29(1):31-49.

Chan, H. C. (1997). Visual query languages for entity relationship model databases. JOURNAL OF NETWORK AND COMPUTER APPLICATIONS, 20(2):203221.

Chavda, M. and Wood, P. (1997). Towards an ODMG-Compliant Visual Object Query Language. In Jarke, M., Carey, M., Dittrich, K., Lochovsky, F., 
Loucopoulos, P., and Jeusfeld, M., editors, Proc. of 23rd International Conference on Very Large Data Bases, pages 456-465.

Chu, W. W., Merzbacher, M. A., and Berkovich, L. (1993). The design and implementation of CoBase. In ACM SIGMOD 93, pages 517-522, Washington D.C.

Chu, W. W., Yang, H., Chiang, K., Minock, M., Chow, G., and Larson, C. (1996). CoBase: A scalable and extensible cooperative information system. Journal of Intelligent Information Systems.

Clark, G. J. and Wu, C. T. (1994). DFQL - Data-Flow Query Language for Relational Databases. INFORMATION AND MANAGEMENT, 27(1):1-15.

Doan, D. K., Paton, N. W., Kilgour, A. C., and al Qaimari, G. (1995). Multiparadigm query interface to an object-oriented database. Interacting with computers, 7:25-47.

Florescu, D., Raschid, L., and Valduriez, P. (1996). A methodology for query reformulation in CIS using semantic knowledge. INTERNATIONAL JOURNAL OF COOPERATIVE INFORMATION SYSTEMS, 5(4):431-467.

Gil, I., Gray, P. M., and Kemp, G. J. (1999). A Visual Interface and Navigator for the P/FDM Object Database. In Paton, N. and Griffiths, T., editors, Proc. User Interfaces to Data Intensive Systems, UIDIS 99, pages 54-63, Edinburgh, Scotland. IEEE Computer Society Press.

Haw, D., Goble, C., and Rector, A. (1994). GUIDANCE: Making it Easy for the User to be an Expert. In Sawyer, P., editor, Proc. 2nd Int. Workshop On Interfaces to Database Systems, pages 19-43. Springer Verlag.

Hripcsak, G., Allen, B., Cimino, J. J., and Lee, R. (1996). Access to data: Comparing AccessMed with query by review. JOURNAL OF THE AMERICAN MEDICAL INFORMATICS ASSOCIATION, 3(4):288-299.

Knuth, D. E. (1968). Semantics of Context-Free Languages. In Mathematical Systems Theory, volume 2, pages 127-145.

Li, W. S. and Shim, J. (1998). Facilitating complex web queries through visual user interfaces and query relaxation. COMPUTER NETWORKS AND ISDN SYSTEMS, 30(1-7):149-159.

Liepins, P. J., Curran, K. M., Renshaw, C. R., and Maisey, M. N. (1998). A browser based image bank, useful tool or expensive toy? MEDICAL INFORMATICS, 23(3):199-206.

Meoevoli, L., Rafanelli, M., and Ricci, F. L. (1994). An interface for the direct manipulation of statistical-data. JOURNAL OF VISUAL LANGUAGES AND COMPUTING, 5(2):175-202.

Merz, U. and King, R. (1994). DIRECT - A Query Facility for Multiple Databases. ACM TRANSACTIONS ON INFORMATION SYSTEMS, 12(4):339-359. 
Murray, N., Goble, C., and Paton, N. W. (1998a). A framework for describing visual interfaces to databases. JOURNAL OF VISUAL LANGUAGES AND COMPUTING , 9(4):429-456.

Murray, N., Paton, N., and Goble, C. (1998b). Kaleidoquery: A Visual Query Language for Object Databases. In Proc. of Advanced Visual Interfaces, pages 25-27, Italy.

Ozsoyoglou, G. and Wang, H. Q. (1993). Example-based graphical database query languages. COMPUTER, 26(5):25-38.

Papantonakis, A. and King, P. (1995). Syntax and Semantics of GQL, a Graphical Query Language. Journal of Visual Languages and Computing, 6:3-25.

Siau, K. L., Chan, H. C., and Tan, K. P. (1992). Visual knowledge query language. IEICE TRANSACTIONS ON INFORMATION AND SYSTEMS, E75D(5):697-703.

Taira, R. K., Johnson, D. B., Bhushan, V., Rivera, M., Wong, C., Huang, L. J., Aberle, D. R., Greaves, M., and Goldin, J. G. (1996). A concept-based retrieval system for thoracic radiology. JOURNAL OF DIGITAL IMAGING, 9(1):25-36.

Yu, C. T. and Meng, W. (1998). Principles of Database Query Processing. Morgan Kaufmann Publishers Inc.

Zhang, G., Chu, W. W., Meng, F., and Kong, G. (1999). Query Formulation from High-Level Concepts for Relational Databases. In Paton, N. and Griffiths, T., editors, Proc. User Interfaces to Data Intensive Systems, UIDIS 99, pages 64-74, Edinburgh, Scotland. IEEE Computer Society Press.

\section{Biographies}

Epaminondas Kapetanios studied statistics and informatics at the University of Athens, Hellas. He postgraduated (M.Sc.) at the Technical University (TH) of Karlsruhe, Germany, and worked as scientific employee at the Research Centre Karlsruhe - Technology and Environment (FZK). Currently, he is working as a research assistant (Ph. D.) at ETH Zurich. His research interests focus on Query Answering Systems and Languages, Semantics, Meta-Data, Ontologies.

Moira C. Norrie is a Professor of Computer Science at ETH Zurich leading a group in the area of Global Information Systems. Her main research interests are in object-oriented and web technologies for information systems.

Dijana Fuhrer-Stakic studied Computer Science at ETH Zurich. In her Diploma thesis, she addressed the visualisation aspects for MDDQL. 